Bryn Mawr College

Scholarship, Research, and Creative Work at Bryn Mawr

College

2019

\title{
Solid state proton spin-lattice relaxation in polycrystalline methylphenanthrenes. IV. 1,4-dimethylphenanthrene
}

Peter A. Beckmann

Bryn Mawr College, pbeckman@brynmawr.edu

Let us know how access to this document benefits you.

Follow this and additional works at: https://repository.brynmawr.edu/physics_pubs

Part of the Physics Commons

\section{Custom Citation}

Beckmann, Peter. 2019. "Solid state proton spin-lattice relaxation in polycrystalline methylphenanthrenes. IV. 1,4-dimethylphenanthrene." Journal of Chemical Physics 150.12: 1-3.

This paper is posted at Scholarship, Research, and Creative Work at Bryn Mawr College. https://repository.brynmawr.edu/physics_pubs/143

For more information, please contact repository@brynmawr.edu. 


\title{
Solid state proton spin-lattice relaxation in polycrystalline methyphenanthrenes IV: 1,4-dimethylphenanthrene.
}

\author{
Peter Beckmann, \\ Department of Physics, Bryn Mawr College, 101 N. Merion Ave, \\ Bryn Mawr, Pennsylvania 19010, USA. \\ Journal of Chemical Physics, 2019, vol 150, paper 124508, pages 1-3. \\ $<\underline{\text { http://doi.org/10.1063/1.5082925 }>~}$
}

\begin{abstract}
We present and model the NMR frequency $(8.50,22.5$, and 53.0 MHz) and temperature (97 to 300 $\mathrm{K})$ dependence of the solid state ${ }^{1} \mathrm{H}$ spin-lattice relaxation process in polycrystalline 1,4dimethylphenanthrene. The solid state gives rise to a situation where methyl group rotation is the only motion on the NMR time scale and the relaxation rates due to the rotations of the 1- and 4methyl groups are conveniently well-separated in temperature. At these low NMR frequencies, both the slow- and fast-motion limits are observed for the rotation of both methyl groups which allows for a more stringent test of the models. The relaxation is nonexponential as expected when it is caused by methyl group rotation in which case the initial relaxation rate is modeled.

Parameters characterizing stretched-exponential fits of the relaxation process are also used both to quantify the degree of nonexponential relaxation and indicate that the observed relaxation is indeed due to the rotation of the two methyl groups. The results are compared with several other polycrystalline methylphenanthrenes and dimethylphenanthrenes. These systems allow for an investigation into how intramolecular and intermolecular interactions between methyl groups and neighboring atoms on the same and neighboring molecules determine the barriers to methyl group rotation.
\end{abstract}

\section{INTRODUCTION}

This is the fourth report on methyl group rotation in solid methyl-substituted phenanthrenes. Here we consider polycrystalline 1,4-dimethylphenanthrene. The compounds dealt with here and in the first three reports ${ }^{1-3}$ are indicated in Table 1 . Methyl group rotation is the only motion on the NMR 


\section{Page 2 of 9}

time scale and the phenanthrene molecule provides an excellent backbone to investigate the intramolecular and intermolecular potentials ${ }^{2-4}$ responsible for barriers to methyl group rotation in these planar aromatic compounds. It is convenient to characterize positions in phenanthrene as $\alpha$, $\delta$, and $\gamma$, as indicated in Fig. 1. For an $\alpha$ methyl group, there is a neighboring ring $\mathrm{H}$ atom on one side on the same ring as the methyl group and a neighboring ring atom on an adjacent ring on the other side of the methyl group. The barriers to methyl group rotation are in the $8-13 \mathrm{~kJ} \mathrm{~mol}^{-1}$ range (Table 1). The differences between methyl group barriers among $\alpha$ methyl groups is partially due to the degree of single versus double bond character in the planar carbon ring structure (intramolecular interactions) and partially due to intermolecular interactions. ${ }^{2,4}$ For a $\delta$ methyl group there are neighboring $\mathrm{H}$-atoms on both sides of the same ring and the barriers are approximately $5 \mathrm{~kJ} \mathrm{~mol}^{-1}$, of which approximately $1 \mathrm{~kJ} \mathrm{~mol}^{-1}$ is due to intramolecular interactions and approximately $4 \mathrm{~kJ} \mathrm{~mol}^{-1}$ is due to intermolecular interactions. ${ }^{4}$ For a $\gamma$ methyl group in the phenanthrene bay region, the rotational barrier is approximately $22 \mathrm{~kJ} \mathrm{~mol}^{-1}$, so long as only the 4 or the $5=4$ position has a methyl group, but not both. For 4,5 -dimethylphenanthrene, which is not included in Table 1, the phenanthrene backbone is greatly distorted and the two methyl group C-C rotation axes are far from the plane on either side of the backbone ${ }^{5}$ leading to methyl group barriers of only approximately $11 \mathrm{~kJ} \mathrm{~mol}^{-1} .{ }^{6}$ For all the systems indicated in Table 1 , the phenanthrene backbone and the rotation axis of the methyl group(s) are in the same plane., ${ }^{3,4}$

\section{THE EXPERIMENTS}

The sample of 1,4-dimethylphenanthrene was purchased from AKos Consulting \& Solutions $\mathrm{GmbH}$ and a high-resolution NMR spectrum showed no impurities at the levels discernable by that technique. The polycrystalline sample was used as is. A (perturbation $\pi$ )-t-(observe $\pi / 2)-t_{\mathrm{w}}$ - pulse sequence was used to perturb and monitor the nuclear magnetization $M(t)$. The wait time $t_{\mathrm{w}}$ was at least ten times the characteristic relaxation time $\left(R^{*}\right)^{-1}$ (discussed below). The experimental technique, including temperature control and measurement, is discussed extensively elsewhere. ${ }^{7}$ The relaxation of the perturbed magnetization $M(t)$ was well characterized by $R^{*}$ and $\beta$ in a stretched exponential [reviewed extensively in ref. 7] $M(t)=M(\infty)+[M(0)-M(\infty)]\left[\exp \left\{-\left(R^{*} t\right)^{\beta}\right\}\right]$ solely to determine the degree of departure from exponential relaxation since $\beta=1$ corresponds to exponential relaxation. Nonexponential relaxation is expected as a consequence of quantum 


\section{Page 3 of 9}

correlations among the three spin- $1 / 2{ }^{1} \mathrm{H}$ nuclei in a methyl group ${ }^{8,9}$ and is well established [Ref. 10 and references therein] when methyl group rotation is responsible for the relaxation. These correlations do not manifest themselves at the beginning of the relaxation process ${ }^{8}$ so the relaxation was also characterized by the short time relaxation rate $R_{\mathrm{S}}$, a parameter that can be modeled. The experimental procedure for determining $R_{\mathrm{S}}$ is outlined in detail elsewhere. ${ }^{7}$ Fig. 2 shows the temperature dependence of $R_{\mathrm{S}}$ at NMR frequencies of 8.50, 22.5, and 53.0 MHz. These low NMR frequencies are needed to see, at temperatures below the melting point, both the hightemperature fast-motion limit $\omega \tau<<1$ and the low-temperature slow-motion limit $\omega \tau>>1$ in order that the data be thoroughly and consistently modeled. Here $\tau$ is the temperature-dependent mean time between methyl group hops (see below) and $\omega /(2 \pi)$ is the NMR frequency. In addition, we show $R^{*}$ for $8.50 \mathrm{MHz}$ to show the relationship between $R_{\mathrm{S}}$ and $R^{*} . R_{\mathrm{S}}>R^{*}$ always and the difference between $R_{\mathrm{S}}$ and $R^{*}$ for 22.5 and $53.0 \mathrm{MHz}$ follows a similar trend to that shown for 8.50 $\mathrm{MHz}$ in Fig. 2. The temperature dependence of $\beta$ is shown in Fig. 3 for $8.50 \mathrm{MHz}$. When $\beta$ is above approximately 0.93 it is difficult to observe the nonexponential relaxation on an experimentby-experiment basis.

\section{RESULTS}

The lower temperature peak in Fig. 2 (initial relaxation rate versus temperature) corresponds to $\omega \tau \approx 1$ where $\tau$ is the mean time between $2 \pi / 3$ hops of the 1-methyl group. The high temperature peak corresponds to $\omega \tau \approx 1$ for the 4-methyl group. It is fortuitous that the two sets of data only slightly overlap while simultaneously providing both the high temperature $(\omega \tau<<1)$ and low temperature ( $\omega \tau \gg>1$ ) regions for $R_{\mathrm{S}}$ versus $T^{-1}$ for both methyl groups. This is usually not the case. ${ }^{1-3}$ Fig. 2 (noting the difference between $R_{\mathrm{S}}$ and $R^{*}$ for $8.50 \mathrm{MHz}$ ) and Fig. $3(\beta)$ together indicate that the greatest departure from exponential relaxation is observed near the maxima in the $R_{\mathrm{S}}$ values and at higher temperatures for both methyl groups. Fig. 3 shows various lines solely to guide the eye for what $\beta$ versus $T^{-1}$ might look like for the 1-methyl group (blue dashed lines) and the 4-methyl group (red dotted lines). The details involve the complexities of the rapid spin diffusion and the $\beta$ values for the two methyl groups do not add algebraically.

We model the relaxation for each methyl group via ${ }^{1} R_{\mathrm{S}}=C[J(\omega, \tau)+4 J(2 \omega, \tau)]$ with $J(\omega, \tau)=(2 / \omega)[\sin \{\arctan (\omega \tau)\}] /\left[\left(1+\omega^{2} \tau^{2}\right)^{\varepsilon / 2}\right], \tau=\tau_{\infty}[\exp \{E / k T\}], \tau_{\infty}=x(2 \pi / 3)(2 I / E)^{1 / 2}$, and 
Page 4 of 9

$C=(n / N)(9 / 40)\left[\mu_{0} /(4 \pi)\right]^{2}\left(\hbar \gamma^{2} / r^{3}\right)^{2} . J(\omega, \tau)$ is the Davidson-Cole spectral density, ${ }^{11} \tau \square$ is the mean time between methyl group hops in a semiclassical (thermally activated) methyl group hopping process for methyl groups in the perfect crystal environment, $\varepsilon$ is a parameter that characterizes a (very small ${ }^{12}$ ) distribution of NMR activation energies $E$ since methyl groups near crystal surfaces or other crystal dislocations may have different barriers than methyl groups in the bulk crystal structure, $I$ is the moment of inertia of a methyl group, $n=3$ is the number of ${ }^{1} \mathrm{H}$ spins in a methyl group, $N=14$ is the number of ${ }^{1} \mathrm{H}$ spins in the molecule, and $r$ is the $\mathrm{H}-\mathrm{H}$ distance in a methyl group. The parameter $C$ can be calculated with no adjustable parameters ${ }^{1,3}$ and accounts for the strength of the ${ }^{1} \mathrm{H}$ spin-lattice relaxation resulting from the modulation of the three pairwise ${ }^{1} \mathrm{H}-{ }^{1} \mathrm{H}$ spin-spin interactions in a methyl group (the intramethyl contribution). The parameter $\tau_{\infty}$ with $x=1$ results from a somewhat simplistic model for the preexponential factor that assumes that between hops the methyl group is vibrating as a harmonic oscillator at the bottom of the rotational potential. ${ }^{13}$ The parameter $x$ is simply a phenomenological fitting parameter that accounts for departure from this simple model. This model, with the parameter $x$, is a useful benchmark and guide. Finally, we note that the NMR activation energy $E$ is closely related to, but not exactly the same as, the barrier $V$ for rotation of a methyl group. The relationship between $V$ and $E$ is complicated and numerical models suggest that $E$ is between 0 and 20\% smaller than $V$ for activation energies $E$ in the range being investigated here. ${ }^{14,15}$ The fitted parameters are $E=12 \pm 1 \mathrm{~kJ}$ $\mathrm{mol}^{-1}, \varepsilon=0.85 \pm 0.08$, and $x=2.0 \pm 0.7$ for the 1-methyl group and $E=22 \pm 2 \mathrm{~kJ} \mathrm{~mol}^{-1}, \varepsilon=0.85 \pm 0.08$, and $x=1.3 \pm 0.6$ for the 4-methyl group. The activation energies and $\varepsilon$ values (determined by the sizes of the crystallites ${ }^{3,10}$ ) can be compared with values for methyl groups on similar positions on the phenanthrene backbone in Table 1.

\section{SUMMARY}

This report concludes our analyses of methyl group rotation in the methylphenanthrene system. The three sets of positions on the planar ring structure where methyl groups can be attached offer a narrow and consistent set of barriers for each position. In the solid state, methyl group rotation is the only motion on the NMR time scale. All this allows for a clean analysis of the relationship between the molecular and crystal structure and the barrier to methyl group rotation. The simple model used here has withstood the test of time. 
Page 5 of 9

${ }^{1}$ K. G. Conn, P. A. Beckmann, C. W. Mallory, and F. B. Mallory, J. Chem. Phys. 87, 20 (1987).

${ }^{2}$ F. B. Mallory, C. W. Mallory, K. G. Conn, and P. A. Beckmann, J. Phys. Chem. Solids, 51, 129 (1990).

${ }^{3}$ P. A. Beckmann, K. G. Conn, C. W. Mallory, F. B. Mallory, A. L. Rheingold, L. Rotkina, and X. Wang, J. Chem. Phys. 139, 204501 (2013).

${ }^{4}$ X. Wang, P. A. Beckmann, C. W. Mallory, A. L. Rheingold, A. G. DiPasquale, P. J. Carroll, and F. B. Mallory, J. Org. Chem. 76, 5170 (2011).

${ }^{5}$ F. Imashiro, A. Saika, and Z. Taira, J. Org. Chem. 52, 5727 (1987).

${ }^{6}$ K. Takegoshi, F. Imashiro, T. Terao, and A. Saika, J. Chem. Phys. 80, 1089 (1984).

${ }^{7}$ P. A. Beckmann and E. Schneider, J. Chem. Phys. 136, 054508 (2012).

${ }^{8}$ R. L. Hilt and P. S. Hubbard, Phys. Rev. 134, A392 (1964).

${ }^{9}$ L. K. Runnels, Phys. Rev. 134, A28 (1964).

${ }^{10}$ P. A. Beckmann, J. Ford, W. P. Malachowski, A. R. McGhie, C. E. Moore, A. L. Rheingold, G. J. Sloan, and S. T. Szewczyk, ChemPhysChem 19, 2423 (2018).

${ }^{11}$ D. W. Davidson and R. H. Cole, J. Chem. Phys. 19, 1484 (1951).

${ }^{12}$ X. Wang, A. L. Rheingold, A. G. DiPasquale, F. B. Mallory, C. W. Mallory, and P. A. Beckmann, J. Chem. Phys. 128, 124502 (2008).

${ }^{13}$ N. L. Owen, in Internal Rotation in Molecules, edited W. J. Orville-Thomas (Wiley, New York, 1974).

${ }^{14}$ J. Kowalewski and T. Liljefors, Chem. Phys. Lett. 64, 170 (1979).

${ }^{15}$ O. Edholm and C. Blomberg, Chem. Phys. 56, 9 (1981).

${ }^{16}$ P. A. Beckmann, Sol. State Nuc. Mag. Resonan. 71, 91 (2015). 
Page 6 of 9

Table 1. NMR activation energies $E$ and distribution parameters $\varepsilon$ for various polycrystalline methylphenanthrenes (MP) and dimethylphenanthrenes (DMP).

\begin{tabular}{|l|l|c|c|c|c|}
\hline molecule & \multicolumn{1}{c|}{$\mathrm{CH}_{3}$} & $\mathrm{CH}_{3}$ & $E$ & $\varepsilon$ & ref \\
\hline & position & type & $\mathrm{kJ} / \mathrm{mol}$ & & \\
\hline & & & $\pm 10 \%$ & $\pm 10 \%$ & \\
\hline 1,9-DMP & 1 & $\alpha$ & 8 & 0.8 & 2 \\
\hline 1,8-DMP & $1,8=1$ & $\alpha$ & 9 & 0.6 & 3 \\
\hline 1,6-DMP & 1 & $\alpha$ & 10 & 0.8 & 3 \\
\hline 1,4-DMP & 1 & $\alpha$ & 12 & 0.9 & this work \\
\hline 9-MP & 9 & $\alpha$ & 11 & 0.6 & 1 \\
\hline 3,9-DMP & 9 & $\alpha$ & 13 & 0.7 & 1 \\
\hline 1,9-DMP & 9 & $\alpha$ & 12 & 0.8 & 2 \\
\hline 3,9-DMP & 3 & $\delta$ & 5 & - & 1 \\
\hline 3-MP & 3 & $\delta$ & 5 & - & 1 \\
\hline 4-MP & 4 & $\gamma$ & 21 & 1.0 & 6 \\
\hline 1,4-DMP & 4 & $\gamma$ & 22 & 0.9 & this work \\
\hline
\end{tabular}


Page 7 of 9

Figure 1

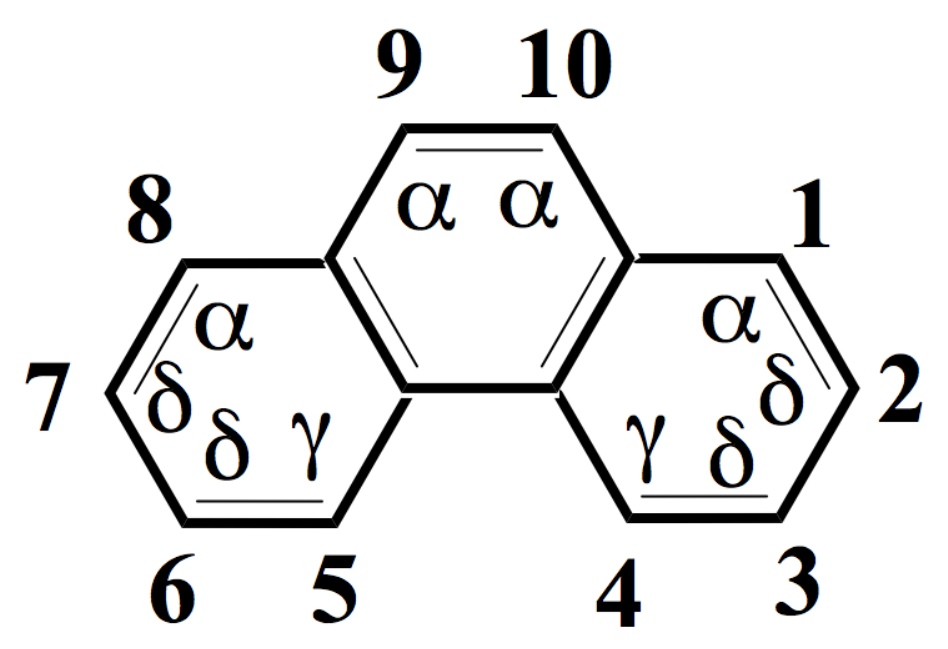

Fig. 1. Methyl group positions on the phenanthrene backbone. $\alpha$ methyl groups have one $H$ neighbor on the same ring and one $\mathrm{H}$ neighbor on an adjacent ring. $\delta$ methyl groups have two $\mathrm{H}$ neighbors on the same ring. $\gamma$ methyl groups are in the bay region. 
Page 8 of 9

Figure 2

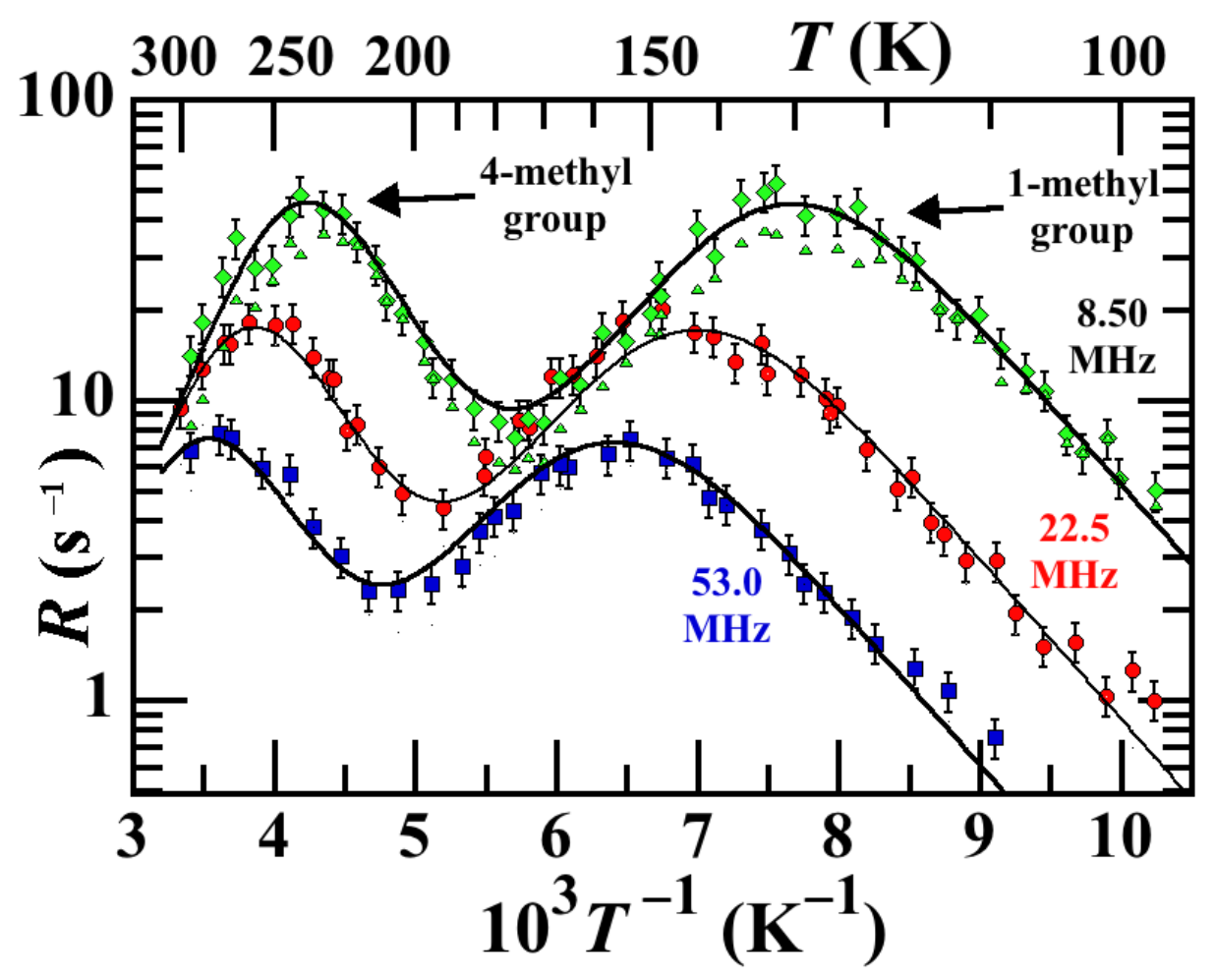

Fig. 2. ${ }^{1} \mathrm{H}$ spin-lattice initial relaxation rates $R_{\mathrm{S}}$ at NMR frequencies of 8.50 (green diamonds), 22.5 (red circles), and 53.0 MHz (blue squares) and the ${ }^{1} \mathrm{H}$ spin-lattice characteristic relaxation rates $R^{*}$ at $8.50 \mathrm{MHz}$ (green triangles) versus inverse temperature $T^{-1}$ for polycrystalline 1,4dimethylphenanthrene. The very small uncertainties in the $R^{*}$ values are approximately the same size as the small green triangles. 
Page 9 of 9

Figure 3

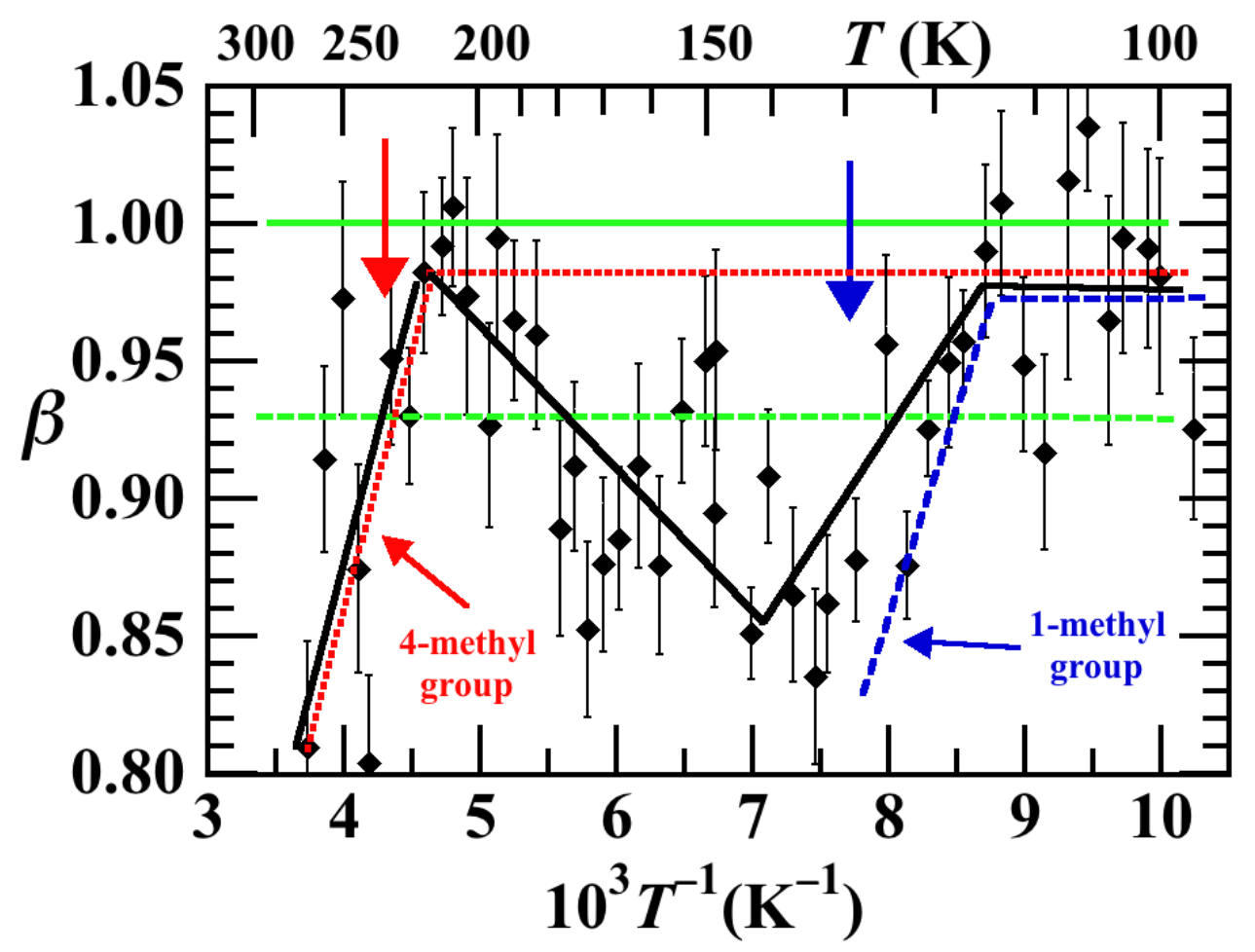

Fig. 3. The stretching parameter $\beta$ in a stretched exponential fit $M(t)=M(\infty)+[M(0)-M(\infty)]\left[\exp \left\{-\left(R^{*} t\right)^{\beta}\right\}\right]$ of the relaxing ${ }^{1} \mathrm{H}$ magnetization $M(t)$ in polycrystalline 1,4-dimethylphenanthrene versus inverse temperature $T^{-1}$ at an NMR frequency of $8.50 \mathrm{MHz} . \beta=1$ (solid green horizontal line) corresponds to exponential relaxation. When $\beta$ is greater than approximately 0.93 (green horizontal dashed line) it is difficult to observe the nonexponential nature of the relaxation on an experiment-by-experiment basis. The downward pointing red arrow at $235 \mathrm{~K}$ and the downward pointing blue arrow at $130 \mathrm{~K}$ indicate the temperatures of the maxima in $R_{\mathrm{S}}$ for the 4- and 1-methyl groups respectively (see Fig. 2). The red dotted lines are, based on experience, ${ }^{16}$ what might be expected for $\beta$ versus $T^{-1}$ if only the 4-methyl group were present and the blue dashed lines are, based on experience, ${ }^{16}$ what might be expected for $\beta$ versus $T^{-1}$ if only the 1-methyl group were present. The solid black lines, which are simply guides for the eye, are the result of the motions of both the 1- and 4-methyl groups. As a consequence of the complexities of spin diffusion, the $\beta$ values do not add algebraically. 